High Resolution Absorption Spectroscopy using Externally Dispersed Interferometry

J. Edelstein, D. J. Erskine

August 12, 2005

SPIE Optics \& Photonics

San Diego, CA, United States

July 31, 2005 through August 4, 2005 
This document was prepared as an account of work sponsored by an agency of the United States Government. Neither the United States Government nor the University of California nor any of their employees, makes any warranty, express or implied, or assumes any legal liability or responsibility for the accuracy, completeness, or usefulness of any information, apparatus, product, or process disclosed, or represents that its use would not infringe privately owned rights. Reference herein to any specific commercial product, process, or service by trade name, trademark, manufacturer, or otherwise, does not necessarily constitute or imply its endorsement, recommendation, or favoring by the United States Government or the University of California. The views and opinions of authors expressed herein do not necessarily state or reflect those of the United States Government or the University of California, and shall not be used for advertising or product endorsement purposes. 


\title{
High Resolution Absorption Spectroscopy using Externally Dispersed Interferometry
}

\author{
Jerry Edelstein ${ }^{a}$ and David J. Erskine ${ }^{b}$ \\ ${ }^{a}$ Space Sciences Lab. at Univ. of Calif., Berkeley, CA 94720-7450 \\ ${ }^{b}$ Lawrence Livermore Nat. Lab., 7000 East Ave, Livermore, CA 94550
}

\begin{abstract}
We describe the use of Externally Dispersed Interferometry (EDI) for high-resolution absorption spectroscopy. By adding a small fixed-delay interferometer to a dispersive spectrograph, a precise fiducial grid in wavelength is created over the entire spectrograph bandwidth. The fiducial grid interacts with narrow spectral features in the input spectrum to create a moiré pattern. EDI uses the moiré pattern to obtain new information about the spectra that is otherwise unavailable, thereby improving spectrograph performance. We describe the theory and practice of EDI instruments and demonstrate improvements in the spectral resolution of conventional spectrographs by a factor of 2 to 6 . The improvement of spectral resolution offered by EDI can benefit space instruments by reducing spectrograph size or increasing instantaneous bandwidth.
\end{abstract}

Keywords: high resolution spectroscopy, externally dispersed interferometer, EDI, resolution boosting, spectral interferometry

\section{INTRODUCTION}

Spectroscopic measurements are central to the future of NASA's mission quests to characterize the surface and atmospheres of astronomical objects; to understand the Sun, stars, and the elements; to study galaxy formation and evolution, and to map the large-scale structure of the universe. We describe a method to advance spectroscopy performance beyond the present bounds of classical instrumentation by using "Externally Dispersed Interferometry" (EDI).

EDI increases an existing grating spectrograph's resolution many fold over its full and simultaneous bandwidth while allowing large etendue and high throughput. Because spatial information is preserved, EDI can be used with slit-imaging, multi-object and echelle spectrometry. EDI uses a series combination of a small fixed delay interferometer with a conventional grating spectrograph. (See Fig. 1-left). Spectral fringes heterodyne fine spectral features into a detectable low frequency moiré pattern. The information thus gained by EDI can be translated directly into improved measurement performance that would be otherwise be limited by the quality of telescopes, or instrumentation. The heterodyning defeats classical performance limits cause by optics blur or the detector pixel Nyquist limit. Furthermore, the precise EDI fringe is a fiducial that can be used to defeat systematic instrumental noise (e.g. detector non-linearity and blur or pupil changes) that limits conventional spectrograph performance.

EDI is a general technique that can enrich the performance of any style of grating or prism spectrograph and is particularly suited for absorption spectroscopy and radial velocimetry measurements. We have demonstrated an EDI resolution-gain of factors of $2-6 \times$ for visible stellar, solar and laboratory measurements taken with echelle and linear grating spectra, e.g. obtaining $\mathrm{R} \sim 140,000$ from an $\mathrm{R}=25,000$ spectrograph. ${ }^{1}$ We have also shown radial velocity measurements with a precision ${ }^{2,3}$ of a few $\mathrm{m} / \mathrm{s}$. Other workers have adopted the EDI method from our laboratories and demonstrated a Doppler planet detection. ${ }^{4}$

While in principle, interferometric spectroscopy can be applied to science instruments for the IR to the X-ray, we concentrate here on applications to optical to far-ultraviolet (FUV) broad-band instruments for absorption

Further information: http://www.spectralfringe.org/EDI

J.E.: jerrye@ssl.berkeley.edu, Tele.: 510-642-0599

D.E.: erskine1@llnl.gov, Tele.: 925-422-9545 
spectroscopy. The EDI resolution gain allows otherwise unattainable instrument profiles that can achieve high performance spectroscopic science, because resolution gain can be traded for parameters such as throughput, physical envelope, mass or for telescope, detector and optics size or tolerance. Likewise, such improvements can provide advanced capability spectrographs for small size or mass limited space platforms.

\subsection{Motivation: The Emerging Universe}

This plan is motivated by the cosmological studies envisioned for a future generation of missions to measure yet undetected baryonic matter, $\geq 25 \%$ of which is postulated to exist as hot and warm gas in cosmic voids (the "cosmic web"). These missions, presently embodied as NASA Origins Mission Concept Studies such as the HORUS $^{5}$ and Baryonic Structure Probe ${ }^{6}$ (BSP), include prime science goals of (1) Measuring the baryonic matter in the universe by surveying the IGM and Lyman- $\alpha$ clouds using absorption spectroscopy of faint (17-20 mag) QSO's. (2) Measuring the evolution of baryonic matter by observing abundance in the Lyman-a clouds and (3) Observing important epochs of galaxy formation. To attaining the science goals, each mission concept concludes that broadband FUV (1025-2000 $\AA$ ) absorption line measurements at high-resolution $(\lambda / \Delta \lambda=R>30 \mathrm{k}$ to $40 \mathrm{k})$ is required, with orders-of-magnitude more sensitivity than the Hubble's STIS or COS instruments. FUV observations are important because this region directly samples resonant transitions from ionized gas at $\mathrm{T}=10^{5}$ to $10^{6} \mathrm{~K}$, gas for which models indicate has a distribution and temporal persistence in the cosmic web which exceeds that of hotter, x-ray absorbing gas by up to a few orders of magnitude.

The HORUS and BSP Mission concepts each include a conventional FUV grating spectrograph. The spectrograph size is large $(1.2-1.5 \mathrm{~m})$ so that the required $40-50 \mathrm{~m} \AA$ resolution can be recorded within at least two (Nyquist limited) minimum size pixels, presumed to be $10 \mathrm{~mm}$ each. The bandpass, spectral resolution and pixel size require $\sim 50 \mathrm{~cm}$ of linear detector recording space. Three separate grating observations (or cross-dispersion) will be needed to observe the full bandpass given the presumed $15-20 \mathrm{~cm}$ detectors. (Note that a $10 \mathrm{~mm}$ pixel represents a yet-unrealized $2.5 \times$ factor of improvement of detector resolution compared to the best existing suitable-format FUV detector i.e. COS/HST).

We offer that the EDI resolution gain can be applied with significant advantage to these Origin's spectrograph concepts. For example, an EDI $2.5 \times$ resolution gain would allow the entire bandpass to be recorded in a single observation at the full resolution by lowering the grating ruling density and dispersion- a remarkable potential gain in mission efficiency. Alternately, the EDI gain can let the required resolution and bandpass be obtained using today's available detectors, or with a telescope or grating that is of $2.5 \times$ worse optical quality than presumed- a remarkable advantage in the tolerance or development risk for the missions.

\section{DISPERSED INTERFEROMETRY}

Classical spectroscopic instruments rely upon the $~ 180$-year old principle of illuminating a diffraction grating with a small aperture and gathering the dispersed light to a sensor. For example, UV-optical high-resolution broad-band space observations have used classical echelle or long-throw grating spectrographs (e.g. Copernicus, IUE, FUSE, HST/STIS, [HST/COS]). The classic principle causes high-resolution spectroscopes to have limited light-gathering power, to be physically large, and to be constrained by sensor capability. Performance is limited by the aperture (light-gathering power) and sensor pixel size because these each directly map to spectral resolution. Physical size is constrained because grating dispersion at the sensor, needed to separate adjacent wavelengths, is proportional to the optical throw-length. The classical approach constrains both large collecting-area observatory-class missions used for photon-starved cosmological investigations and missions with limited-size probes. By the laws of optical conservation, classical spectrographs used with large telescopes must scale to large instruments with exacting mechanical requirements while small instruments struggle to obtain adequate spectral dispersion.

\subsection{EDI}

The externally dispersed interferometer ${ }^{2,3,7,8}$ is a general technique that can enhance the performance of any style of grating or prism spectrograph used for spectroscopy or Doppler velocimetry. The EDI can multiply the spectral resolution and increase the Doppler sensitivity of an existing spectrograph over the spectrograph's full and simultaneous bandwidth by a factor of several to an order of magnitude while preserving imaging. ${ }^{9}$ For 

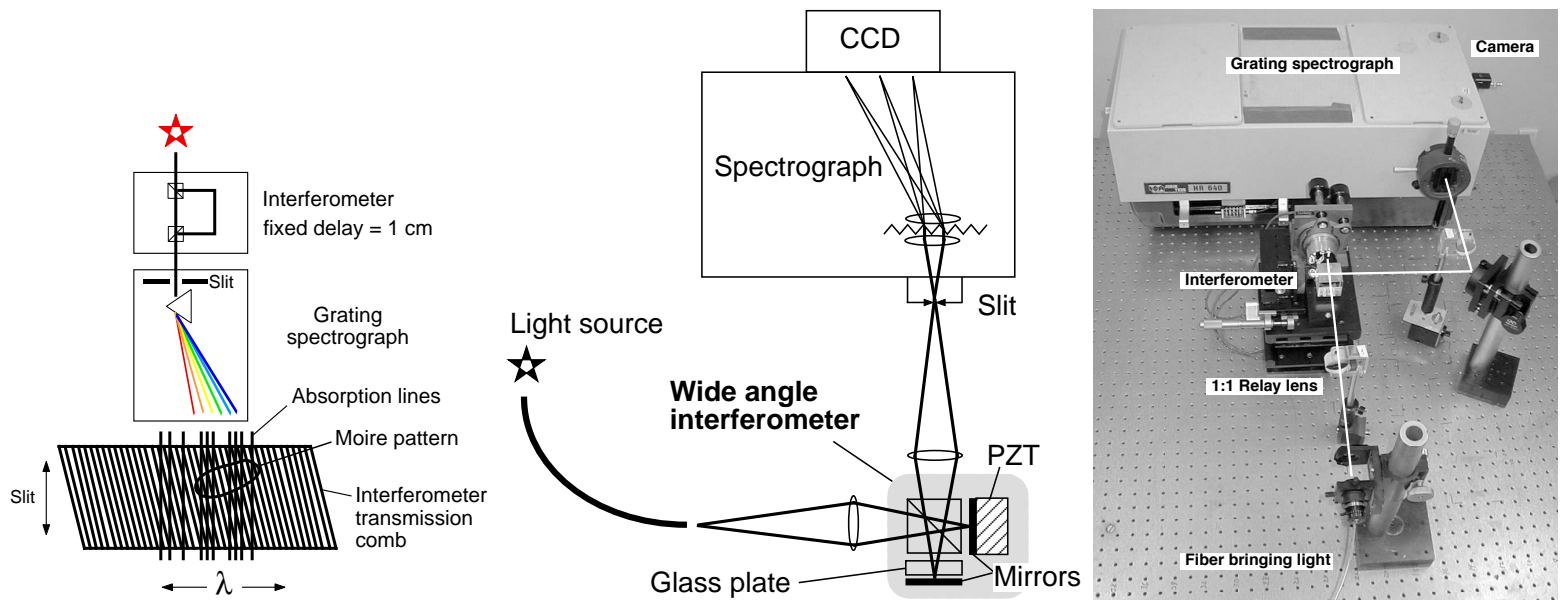

Figure 1. (Left) Principle of EDI: A white-light, unequal-arm interferometer feeds a spectrograph and produces spectral fringes that beat with the source's spectrum and create a recordable moiré pattern. (Mid \& right) Laboratory EDI spectrograph. Light, after traversing an optional calibrating absorption cell, traverses a small, unequal-arm, field-widened Michelson interferometer and is focussed onto the slit of a conventional grating spectrograph. This instrument used a $\mathrm{R}=20 \mathrm{k}$ spectrograph and $\tau=1 \mathrm{~cm}$ delay interferometer to achieve an effective resolution of $\mathrm{R} \sim 50 \mathrm{k}$ and was used to detect the $12 \mathrm{~m} / \mathrm{s}$ Doppler signature in sunlight due to the Moon's gravity on Earth. Neither thermal nor convection control were used. The 1" sized optics were commercial moderate quality components.
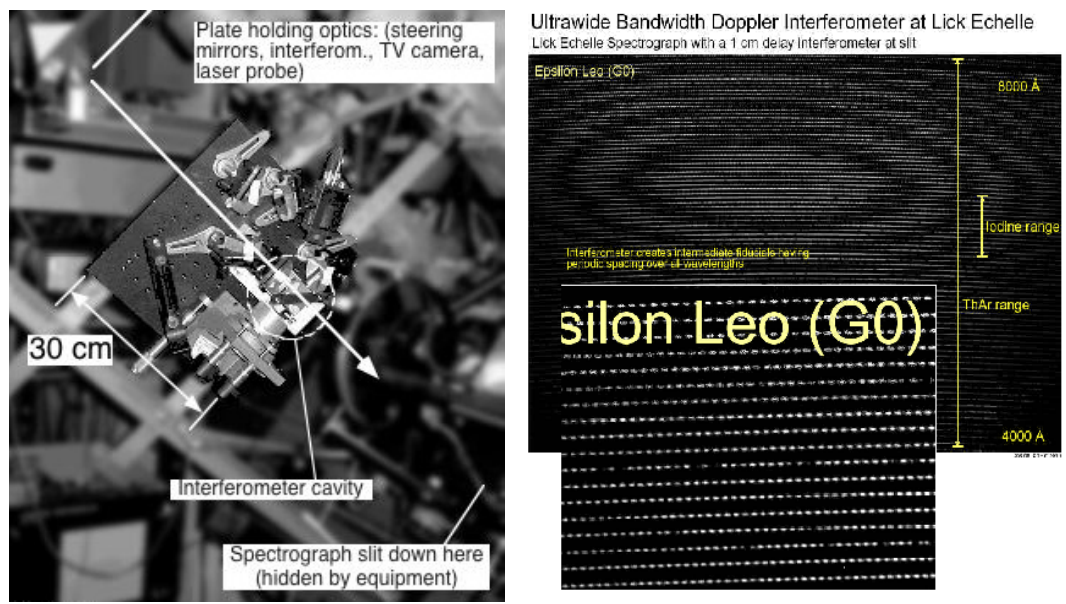

Figure 2. A visible light EDI interferometer (left) installed at the Lick Observatory coudé echelle spectrograph. Starlight from the telescope passes through the $\lambda / 8$ stabilized interferometer, shown mounted on its $30 \mathrm{~cm}$ base plate. Interferometer fringes are imaged to the spectrograph's entrance slit by a lens (these are hidden by equipment) and dispersed by the echelle to a CCD detector. The raw stellar EDI data (right) of $\epsilon$-Leo shows the bead-like fringing pattern in each order beating with absorption lines in the spectrum (see inset detail of orders). 
example, our prototype observatory and laboratory instruments have demonstrated up to a factor of six increase ${ }^{1}$ in conventional visible-band spectrograph resolving power (from $\mathrm{R}=25 \mathrm{k}$ to $\mathrm{R}=140 \mathrm{k}$, and fringe-shift sensitivity of $\lambda / 15,000$ and Doppler shifts precision ${ }^{8}$ of a few $\mathrm{m} / \mathrm{s}$.

EDI uses a series combination of a small fixed delay interferometer of moderate precision $(\lambda / 4-\lambda / 8)$ with a conventional grating spectrograph (see Figs. 1). The interferometer can be inserted and removed like a filter from the input beam without effecting the existing spectrograph. The interferometer produces stable fiducial spectral fringes (Fig. 2), sinusoidal in wavenumber $(\nu=1 / \lambda)$, that heterodyne fine spectral features into a detectable low frequency moiré pattern at the spectrograph sensor (Fig. 3-left). The heterodyning can be numerically reversed to recover detailed spectral information otherwise unattainable by the spectrograph alone. In EDI, the moiré fringe phase becomes the primary diagnostic instead of spectral dispersion. Since these shifts are nearly the same over the entire bandwidth, they can be averaged together to produce a strong net signal. The moiré patterns rotate in phase versus the Doppler shift $\Delta v_{D}$ as $e^{i 2 \pi \tau \Delta v_{D}}$ where $\tau$ is the delay in the interferometer arms.

The EDI heterodyning phenomena overcomes classical-spectrograph constraints on resolution performance, caused by the slit width and detector pixel size, over the spectrograph's entire simultaneous bandpass whereas conventional high-resolution spectrographs are severely limited in bandpass. The EDI fringing signal provides a precise internal fiducial that can be used to defeat systematic instrumental noise and obtain robust spectral performance with a large tolerance to blur or pupil changes. ${ }^{10}$ This performance stability differs from classical high-resolution spectrographs that directly map telescope image quality and pupil stability loss to spectral degradation.

The EDI peak response to spatial frequencies is set by the interferometer delay. The EDI response can be selected to optimize sampling of the desired science spectral signature and to measure high spatial frequencies far beyond the spectrograph's resolution limit. An important property of EDI is that the most stable region of the grating spectral response is shifted from low spatial frequencies in the spectrum to frequencies where the desired science information resides. Consequently, the EDI science performance is highly tolerant of changes of the grating's conventional spectral profile or of optics pupil changes.

EDI and its application to absorption spectroscopy are fundamentally distinct from other interferometric spectrometers. Only EDI is capable of broad-band absorption spectroscopy with signal-to-noise performance that is on par with classical grating instruments. EDI differs from previous heterodyning hybrids (e.g. SHS \& HHS $^{11-13}$ ) by using an external, rather than internal dispersing element. Internal dispersion severely limits bandwidth because the SHS fringe spacings quickly exceed detector resolution. EDI dispersion dramatically mitigates the wide-band continuum noise penalty suffered by internally-dispersed interferometers for absorption measurements. Compared to a dispersed Fabry-Perot interferometer that produce spike-like spectral fiducials, ${ }^{14,15}$ EDI's sinusoidal fringes transmit more flux and allow simple Fourier reduction schemes and an elegant 3-phase trigonometric recovery of spectral information. Compared to Fourier Transform Spectroscopy (FTS), EDI offers a simultaneous bandpass and $100 \times$ signal-to-noise improvements for absorption measurements.

\section{EDI PRINCIPLES, PERFORMANCE}

\subsection{Demonstrations \& Principles}

We have demonstrated prototype laboratory and observatory EDI instruments for broad visible band highresolution absorption spectroscopy ${ }^{9,10}$ and radial velocity measurements of starlight ${ }^{8}$ and sunlight, ${ }^{2}$ as well as development of theory and data-reduction algorithms. Doppler velocity EDI measurements ${ }^{2,3,7,8}$ detected the $12 \mathrm{~m} / \mathrm{s}$ amplitude of the Moon tugging the earth with an estimated long-term instrument error of $4 \mathrm{~m} / \mathrm{s}$ and short term precision of a $(\lambda / 15,000)$ fringe shift. (This work was funded by prior LLNL projects for ground-based Doppler velocimetry planet searches.)

We illustrate EDI principles and performance through our demonstrations. Stellar and solar EDI spectra were acquired using the full bandwidth of 4200-8000 $\AA$ of the Lick Observatory's Hamilton 2-d echelle spectrograph. ${ }^{16}$ The prototype EDI interferometer (Fig. 2) has a compact $30 \mathrm{~cm}$ footprint, uses moderate $(\lambda / 4)$ quality 1-inch size optics and a PZT cavity stabilizer. The apparatus demonstrates the EDI's resolution performance and was not optimized for sensitivity- we discarded one interferometer arm output, a $50 \%$ loss. (We later describe EDI's using both arm outputs.) Reduced EDI spectra of the Sun and $\alpha$-Virgo are shown in Figs. 26 and 28 (and 

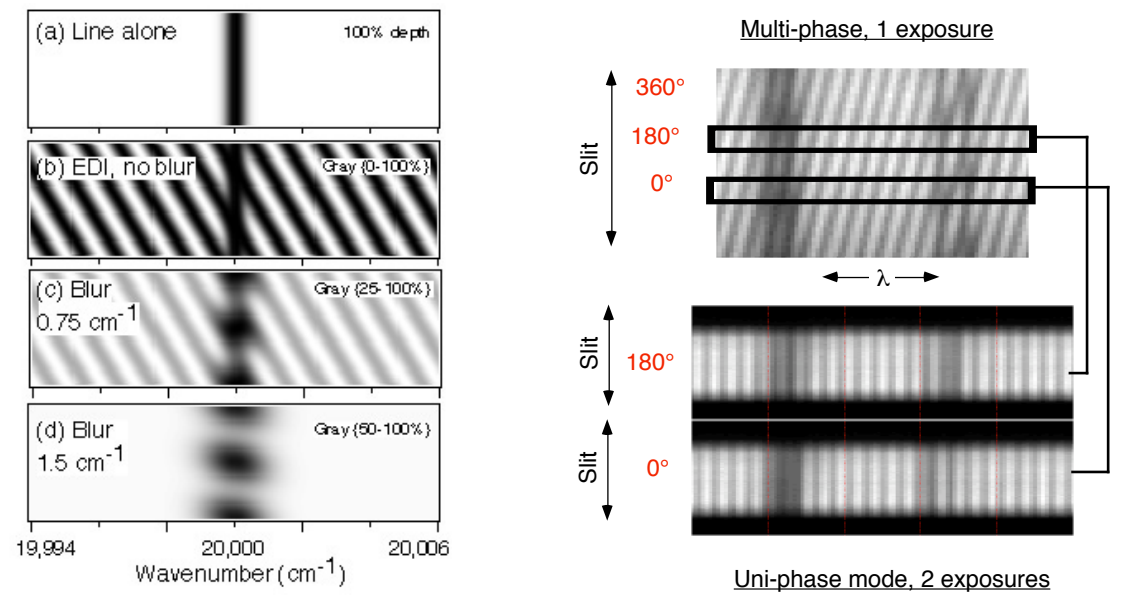

Figure 3. (Left) EDI moiré pattern. A spectral line (a) is multiplied by (slanted) EDI fringes in the spectral or horizontal axis (b). The varying fringing phase beats with the spectral line to form a moiré that persists when the spectral resolution is blurred (c) to the point that the EDI fringes themselves can no longer be resolved (d). (Right) Multi-phase (a) and uni-phase (b) recording. In multi-phase EDI all interferometer phases are recorded in a single exposure because the fringe phase is varied (slanted) across the spectrograph slit. In uni-phase recording the fringes are uniform over the slit and different phases are recorded in different exposures. Uni-phase recording with zero slit-height allows for imaging and echelle spectroscopy.

are discussed further below). These demonstrate a $2-2.5 \times$ resolution gain, i.e. $\mathrm{R}=100,000$ using a $\mathrm{R}=50,000$ conventional spectrograph.

\subsection{Uniform, Multi \& Stepped Phase}

Our prototype Lick interferometer used a wide angle Michelson design ${ }^{17}$ having non-zero optical path length difference (delay, $\tau$ ). Small delay changes $(\delta \tau)$ are described by an interferometer phase $(\phi=2 \pi \delta \tau / \lambda)$. Data can be taken in either of two modes where $\phi$ is either uniform (uni-phase) or varying (multi-phase) along the slit length. In the multi-phase mode, an interferometer mirror is tilted so that $\phi$ varies (Fig. 3a-right) so the full range of $\phi$ can be sampled in a single exposure. In the uni-phase fringe mode, the interferometer mirrors are parallel, $\phi$ is made constant over the slit, and a minimum of three phase-stepped exposures are acquired. The uni-phase mode (Fig. 3b-right) can be used with zero slit-length which maximizes the signal to detector-background ratio and permits EDI application to 1-d imaging or echelle spectroscopy. Using phase-stepped exposures strongly rejects systematic noise (e.g. fixed-pattern detector distortions or optical scatter) that corrupts conventional spectrograph performance.

\subsection{EDI Theory: Fringe Formation}

The conventional (purely dispersive spectroscopy) detected signal versus wavenumber, $B_{\text {ord }}(\nu)$, is the convolution of the intrinsic input spectrum, $S_{0}(\nu)$, and the spectroscope line spread function $\operatorname{LSF}(\nu)$,

$$
B_{\text {ord }}(\nu)=S_{0}(\nu) \otimes L S F(\nu) .
$$

The convolution of Eq. 1 is conveniently expressed in Fourier-space as a product,

$$
b_{\text {ord }}(\rho)=s_{0}(\rho) l s f(\rho)
$$

where lower case symbols are the transforms, and $\rho$ is the spatial frequency along the dispersion axis in features per $\mathrm{cm}^{-1}$. The $\operatorname{lsf}(\rho)$ is thus the transfer function of the impulse response $\operatorname{LSF}(\nu)$. The normalized interferometer transmission $T^{\prime}(\nu)$ is a sinusoidal spectral comb,

$$
T^{\prime}(\nu)=1+\gamma \cos (2 \pi \tau \nu+\phi)
$$


where $\gamma$ is the interferometer visibility, assumed unity for now, and $\tau$ and $\nu$ in units $\mathrm{cm}$ and $\mathrm{cm}^{-1}$, respectively. Raw fringing spectra $B_{\phi}$ are recorded at multiple phase values $\phi$ differing by $\sim 90^{\circ}$, designated $B_{0}, B_{90}$, etc. The passage of light through the interferometer multiplies the spectral comb $T^{\prime}(\nu)$ with the spectrum prior to blurring by the external spectrograph. Hence the EDI detected signal is

$$
B_{\phi}(\nu)=\left[S_{0}(\nu) T^{\prime}(\nu)\right] \otimes L S F(\nu) .
$$

This is re-expressed as a sum of the ordinary spectrum plus two complex counter-rotating fringing components:

$$
B_{\phi}(\nu)=B_{\text {ord }}(\nu)+\frac{1}{2}\left[S_{0}(\nu) e^{i \phi} e^{i 2 \pi \tau \nu}+S_{0}(\nu) e^{-i \phi} e^{-i 2 \pi \tau \nu}\right] \otimes L S F(\nu) .
$$

High-resolution information is recovered by isolating a fringing component and reversing the heterodyning. The scalar spectrum is converted to a complex vector spectrum called a "whirl", W( $\nu)$, by using a linear combination of the phased exposures $B_{\phi}$ with the phases numerically synchronized to each exposure's phase-step value. The whirl for a four phase recording every $90^{\circ}$ is

$$
\mathbf{W}(\nu)=\frac{1}{2}\left(B_{0} e^{i 0^{\circ}}+B_{90} e^{i 90^{\circ}}+\ldots\right)
$$

or

$$
\mathbf{W}(\nu)=\frac{1}{4}\left[\left(B_{0}-B_{180}\right)+i\left(B_{90}-B_{270}\right)\right]
$$

The whirl is then:

$$
\mathbf{W}(\nu)=\frac{1}{2}\left[S_{0}(\nu) e^{i 2 \pi \tau \nu}\right] \otimes L S F(\nu) .
$$

and the Fourier transform of the whirl is

$$
\mathbf{w}(\rho)=\frac{1}{2} \gamma s_{0}(\rho+\tau) \operatorname{lsf}(\rho)
$$

now including interferometer visibility $(\gamma)$.

This important equation describes the EDI formation of moiré fringes, a heterodyning effect expressed in the $s_{0}(\rho+\tau)$ argument. Fine spectral details having high feature density $\rho$ are heterodyned (shifted by $\tau$ ) to measurable low density, $\rho$ features prior to any blurring by the spectrograph's line spread function. The optimal delay value of $\tau \sim 1 / \delta \nu=R / \nu$ will shift the heterodyned fringing response peak to the shoulder of the ordinary response peak. For example $\tau=1 / 2 \mathrm{~cm}$ for a $\mathrm{R}=30,000$ grating at $\nu=60,000 \mathrm{~cm}^{-1}(1667 \AA)$.

The ordinary spectrum is determined by summing the phase-stepped data so that fringing terms cancel,

$$
B_{\text {ord }}(\nu)=\frac{1}{4}\left(B_{0}+B_{180}+B_{90}+B_{270}\right) .
$$

\subsection{Spectral Recovery and Response}

To obtain the EDI spectra, the optical heterodyning is numerically reversed and the ordinary and fringing spectral components are combined. This is analogous to combining the "treble" and "bass" channels of an audio system to obtain a more full bodied sound. The bass channel is the ordinary spectrum (the simple grating-spectrograph recording) and the treble channel is the fringing spectrum that contains new high resolution information. A demonstration of the EDI method is shown in Fig. 4-left.

The ordinary spectrum is found by summing the data, as fringing terms cancel. To recover high-frequency spectral components, we translate $\mathbf{w}(\rho)$ by $\tau$ and scale it by $2 / \gamma$. We form the EDI "treble" Fourier signal $b_{\text {edi }}^{\prime}(\rho)$ by mirroring and conjugating the positive frequency branch so that both branches represent a Fourier transform of a purely real spectrum.

The net Fourier signal, $b_{n e t}(\rho)$, is formed from the fringing and ordinary signals:

$$
b_{n e t}(\rho)=\frac{\alpha(\rho) b_{\text {ord }}(\rho)+\beta(\rho) b_{\text {edi }}^{\prime}(\rho)}{\alpha(\rho)+\beta(\rho)} .
$$



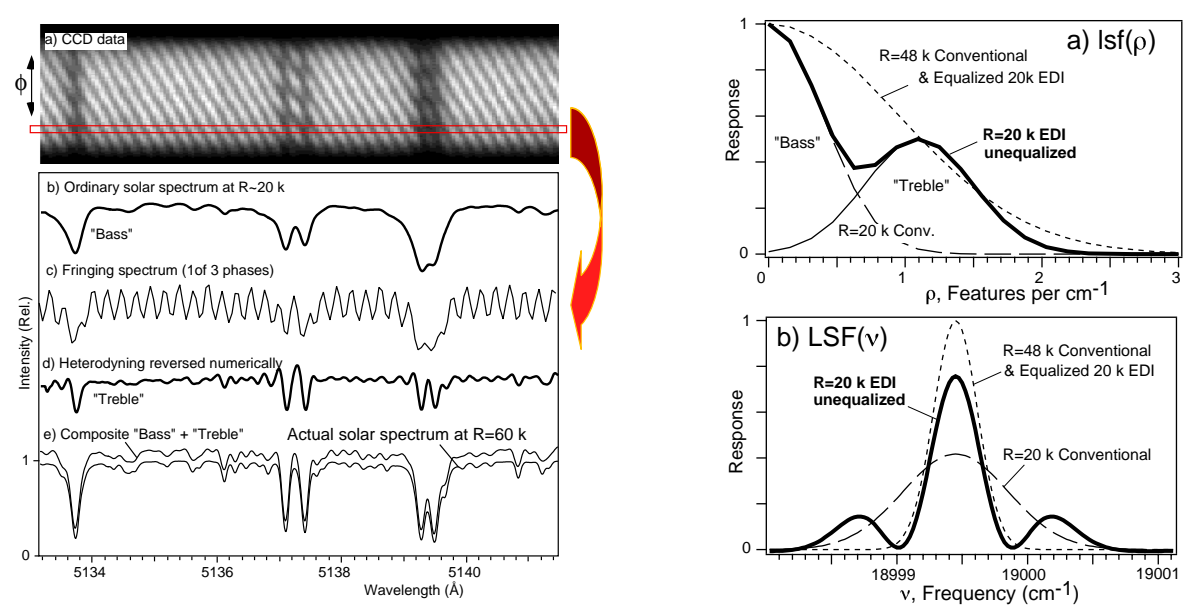

Figure 4. (Left) EDI data reduction illustrated for a solar spectrum using a R $20 \mathrm{k}$ conventional spectrograph and a $\tau=1.1 \mathrm{~cm}$ delay interferometer. (a) The detector image of the multiphase EDI data where $\phi$ varies transversely (vertical) to dispersion (horizontal). (b) The ordinary spectrum from the total histogram sum of (a). (c) A phase recording of the fringing spectrum selected from a limited $\phi$ range of (a) (d) The fringing spectrum made from a combination of phase recordings. (e) The EDI composite spectrum (lower curve) including equalization is nearly identical to a reference Kitt Peak FTS solar spectrum (upper curve) numerically blurred to $\mathrm{R}=60,000$. This EDI yields a $2.5 \times$ resolution gain. (Right) (a) EDI spectral response functions for the ordinary and fringing spectral components vs. spectral feature frequency, $\rho$. The ordinary response ("Bass") is Gaussian at $\rho=0$ shown for a $\mathrm{R}=20 \mathrm{k}$ grating. The EDI fringing response ("Treble") is the ordinary response shifted by the delay $\tau \sim 1.2 \mathrm{~cm}$ and scaled by the visibility/2. The net spectral response (bold) is similar to the response for an $\mathrm{R}=48 \mathrm{k}$ grating (short dash), indicating the EDI increases resolution $2.4 \times$. (b) The EDI spectral line profile (bold), unequalized, is similar to an $\mathrm{R}=48 \mathrm{k}$ grating profile (short dash). The "ringing" side-bands are stable and can be precisely suppressed using equalization by compensating for the relatively diminished $\rho \sim 0.7 \mathrm{~cm}^{-1}$ response region seen in (a). The equalized line profile is Gaussian. See Fig. 5-right for an example line profile after equalization.

The masks $\alpha$ and $\beta$ are chosen to avoid noise contribution from spectral regions with insignificant signal. Optimal $\mathrm{S} / \mathrm{N}$ occurs when $\alpha$ and $\beta$ are proportional to the ordinary and fringing response functions, respectively. Finally, the real spectrum, $B_{n e t}(\nu)$, is obtained from the inverse transform of $b_{\text {net }}(\rho)$.

The theoretical EDI Fourier response functions $\left|b(\rho) / s_{0}(\rho)\right|$ for ordinary and fringing components is shown in Fig. 4a-right. The ordinary response is modeled by a Gaussian $l s f(\rho)$ centered at $\rho=0$ with a width corresponding to the conventional grating. The EDI-response is $l s f(\rho)$ shifted by the delay $\tau$ and scaled by $\gamma / 2$. The net EDI response $\left|b_{n e t}(\rho) / s_{0}(\rho)\right|$, after normalization to constant photon noise, is approximately the quadrature combination of the ordinary and fringing terms and, for the high resolution information found at high $\rho$, follows a conventional grating response with a factor of several higher resolution than the original ordinary grating. An unequalized EDI spectral line profile $\operatorname{LSF}(\nu)$ is shown in Fig. 4b-right.

The EDI profile is similar to that of a higher resolution spectrograph. Because EDI gains more high than mid frequency information (see the negative inflection in Fig. 4a-right) line-profile "side-bands" appear. The side-bands are stable and can be precisely suppressed using apodization (equalization) methods familiar to radio-astronomy to obtain a Gaussian profile. Calibration spectra establish the equalization component. We demonstrate the quality of equalized EDI spectral response for genuine and simulated data in Fig. 4-left, 5-left \& 5-right.

\subsection{Sensitivity}

EDI sensitivity is, in general, comparable to that of classical spectrographs. The EDI can be arranged to record both complementary interferometer beam-splitter outputs (see later) so that all the photons are recovered. Detrimental factors include throughput loss due to added interferometer optical elements. These losses are recovered by making use of the increased slit-acceptance or bandpass now allowed by the instrument. Dividing 

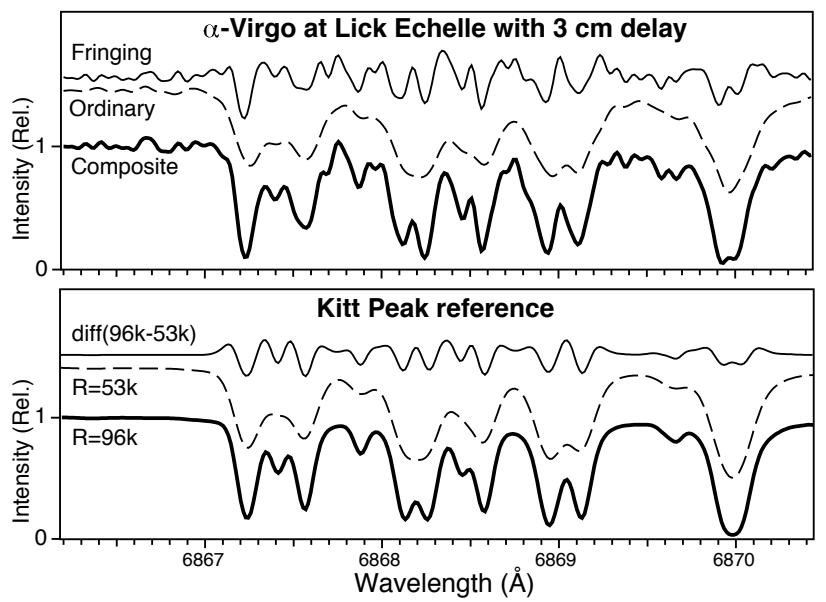

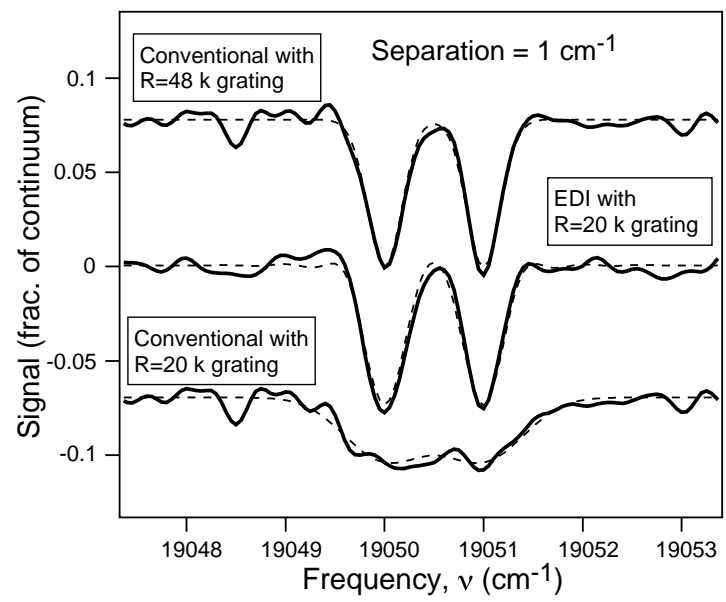

Figure 5. (Left) EDI spectra of O telluric lines in $\alpha$-Virgo using the Lick echelle (conventional R=50 k). Top: The EDI measured ordinary spectrum (dashed line) and fringing spectrum (thin line) are combined to form the composite spectrum (bold line). Bottom: A reference solar spectrum including the telluric lines, blurred to $\mathrm{R}=53 \mathrm{k}$ (dashed line), $\mathrm{R}=96 \mathrm{k}$ (bold), and their difference (thin) compare very closely to the EDI results. The differences are consistent with statistical noise. (Right) Simulation comparing resolution of an absorption doublet by a conventional $\mathrm{R}=48 \mathrm{k}$ spectrograph (upper), an EDI with $\mathrm{R}=2.4 \times 20 \mathrm{k}$ (center), and conventional $\mathrm{R}=20 \mathrm{k}$ spectrograph (lower). The instrument profiles of the EDI and the $\mathrm{R}=48 \mathrm{k}$ spectrographs are similar. Although the same detector noise field was used for each case, the EDI noise looks different because heterodyning and equalization causes a relative enhancement of continuum noise at certain spatial frequencies. The doublet is Gaussian, at $5250 \AA$ with $50 \%$ depth, $17 \mathrm{~m} \AA$ width and separated by $0.26 \AA$. Photon noise is included (bold) at $1.24 \%$ of the continuum 6 pixel per $\mathrm{cm}^{-1}$. Dashed curves are noiseless data.

the observations into phase-stepped recordings does not in itself loose flux because the phases are added to recover the ordinary and fringing spectra. Noise in the data will propagate through the Fourier data analysis and manifest errors in the derived fringing spectra. One of our prime objectives is to derive, illustrate and verify exactly how signal-to-noise manifests itself in pragmatic spectral observables under different conditions.

\subsection{EDI Statistical Noise Theory}

The EDI photon noise per spectral interval, $\sigma_{B(\nu) n e t}$, in the combined EDI spectra is determined by the noise in the ordinary and fringing spectral components. The noise for the ordinary component is the same (in both $\nu$ and $\rho$-space) as that for the conventional spectrograph for the same total exposure time because each phase-recording $B_{\phi}$ is an independent measurement.

The noise for the EDI and ordinary components are similar because the EDI component (Eq. 6) is constructed of the same $B_{\phi}$ terms as the ordinary component differing only by unity magnitude phasors. The phasors do not affect the net noise statistically because the $B_{\phi}$ are independent and the phasors only rotate $B_{\phi}$. Random noise in the spectrum $B(\nu)$ produces uniform, random noise in $b(\rho)$ hence, the same noise magnitude per $\rho$-bin exists for both $b_{\text {ord }}(\rho)$ and $b_{e d i}^{\prime}(\rho)$. Furthermore, the noise per $\rho$-bin in $b_{\text {net }}(\rho)$ is the same as for its components $b_{\text {ord }}$ and $b_{e d i}^{\prime}$ because the net response is formed from a weighted composite that effectively includes only one of the two components at each $\rho$. Therefore, before equalization, $\sigma_{B(\nu) n e t} \approx \sigma_{B(\nu) c o n v} \approx \sigma_{B(\nu) E D I}$.

Equalization will affect noise in the final spectrum. The equalization process scales the photon noise for only the mid- $\rho$ "notch" region. Therefore the noise in the resulting spectrum for the high- $\rho$ (high spectral resolution features) is not affected while the noise for mid- $\rho$ features will be scaled by a factor $U$, the ratio of equalized to unequalized responses at mid- $\rho$. The value of $U$ will depend upon $\tau / R$ and $\gamma$.

We illustrate the photon noise effects present in EDI spectra taken with our prototype EDI by using a low flux measurement (with significant statistical noise) of telluric absorption lines in $\alpha$-Virgo. The measured EDI spectrum is shown in Fig. 5-left together with a reference spectrum for comparison. In this case, from the spectral response function for broad $(\rho<1 \mathrm{~cm})$, midrange $(\rho \sim 2 \mathrm{~cm})$, and narrow $(\rho>3 \mathrm{~cm})$ spectral feature size, this 

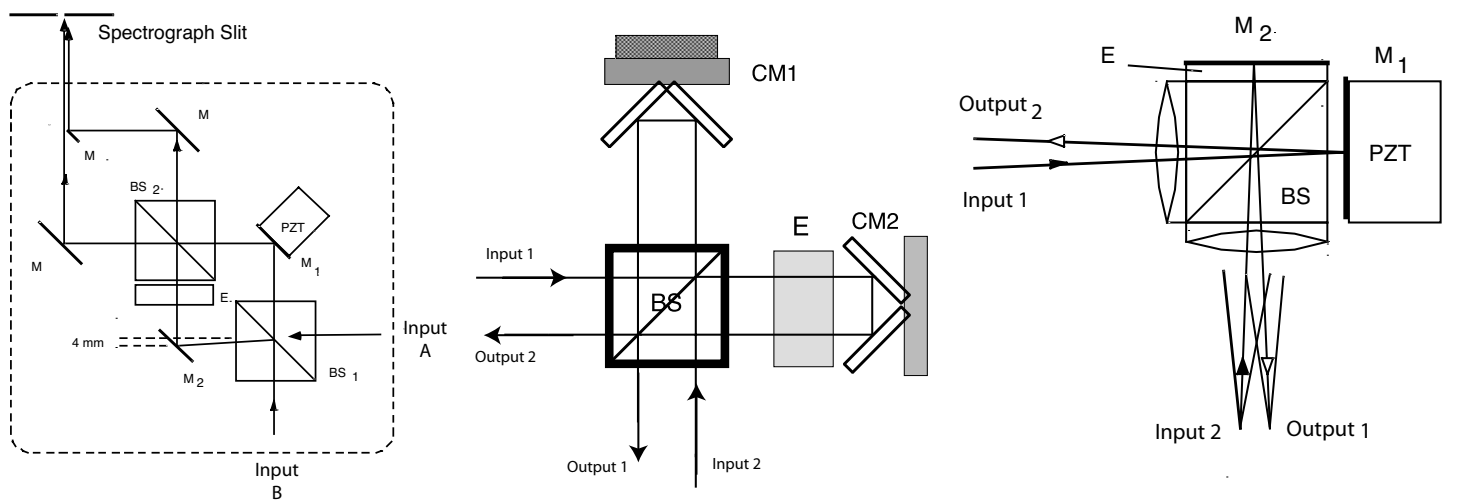

Figure 6. Complementary Output Interferometers - Schemes show designs for obtaining the interferometers' complementary outputs so that full energy is recovered. (and provide complementary inputs). Complementary inputs also exist that can be used for differential spectroscopy. All designs can use a thin-plate beam-splitter, in place of the thick cube shown (BS), for high FUV transmission.

EDI instrument's predicted net continuum to noise ratio $(\mathrm{C} / \mathrm{N})$ is $\sim 80 \%, \sim 50 \%$, and $\sim 100 \%$ of the conventional $\mathrm{C} / \mathrm{N}$ for a $\mathrm{R}=100,000$ grating, respectively. The $\mathrm{C} / \mathrm{N}$ for both the conventional and the unequalized net EDI spectra is $\sim 37$ at 18 pixel per $\AA$, or $\sigma_{B(\nu) c o n v} \sim \sigma_{B(\nu) \text { net }} \sim 3 \%$. For the final equalized net EDI spectrum $\mathrm{C} / \mathrm{N} \sim 20$ or $\sigma_{B(\nu) \text { equalized }} \sim 5 \%$. The differences between the reference and EDI spectrum in the result are consistent with the statistical noise in the data recording.

In Fig. 5-right we show numerical simulations illustrating the effect of random statistical noise on conventional $\mathrm{R}=20 \mathrm{k}, \mathrm{R}=48 \mathrm{k}$ and $\mathrm{EDI} \mathrm{R}=48 \mathrm{k}(\mathrm{R}=20 \mathrm{k} \times 2.4$ resolution gain $)$ spectra. Although the EDI equalization process enhances continuum noise at certain spectral feature frequencies, the simulation shows differences between the EDI and conventional $\mathrm{R}=48 \mathrm{k}$ spectra that are just perceptible at the scale of a spectral feature.

\section{ASPECTS OF EDI TECHNOLOGY}

\subsection{EDI Analysis \& Simulation}

We have developed a set of software to reduce EDI data. The code reduces a set of phase stepped exposures into a complex spectrum, eliminates the fringing component to form a conventional spectrum as an intermediate output, performs heterodyning reversal, and computes equalization and the final resolution-boosted composite spectrum. This research software will be improved for speed, ease of use, and accuracy.

\subsection{Cavity Stabilization}

Interferometer fringes recorded during an integrating exposure requires a stable optical path phase difference, $\phi$. If $\phi$ wanders by $\sim \lambda / 4$, then the net visibility $\gamma$, and proportionally $\mathrm{S} / \mathrm{N}$, will be notably reduced. Optical paths can be changed by thermal dimensional changes and short-term vibrations. We use a commercial piezo mounted mirror and custom software to actively compensate for optical path fluctuations. A visible laser propagates parallel to ("piggy-back") the science beam via the same interferometer elements to form fringe patterns on a small CCD camera. Real-time software analyzes the location of the fringe pattern to compensate for motion. The same piezo-system is used to step $\phi$ for phase step recordings. We have implemented stabilization for the critical "piston" mirror motion and will extend the stabilization system to also compensate for beam tip or tilt.

\subsection{Configurations \& Complementary I/O}

To obtain full efficiency from the EDI, both interferometer complementary outputs need to be collected. Various complementary output designs that have been functionally verified in our laboratory are shown in Fig. 6 . In the Mach-Zehnder (M-Z) scheme (a), the outputs are conveniently separated and can be collected to either a different spectrograph, or joined along a single spectrograph's imaging slit. $\mathrm{M}_{1}$ is piezo actuated for phase stabilization and stepping. Note that the complementary outputs are $180^{\circ}$ out of phase so only a single $\lambda / 2$ path step yields 

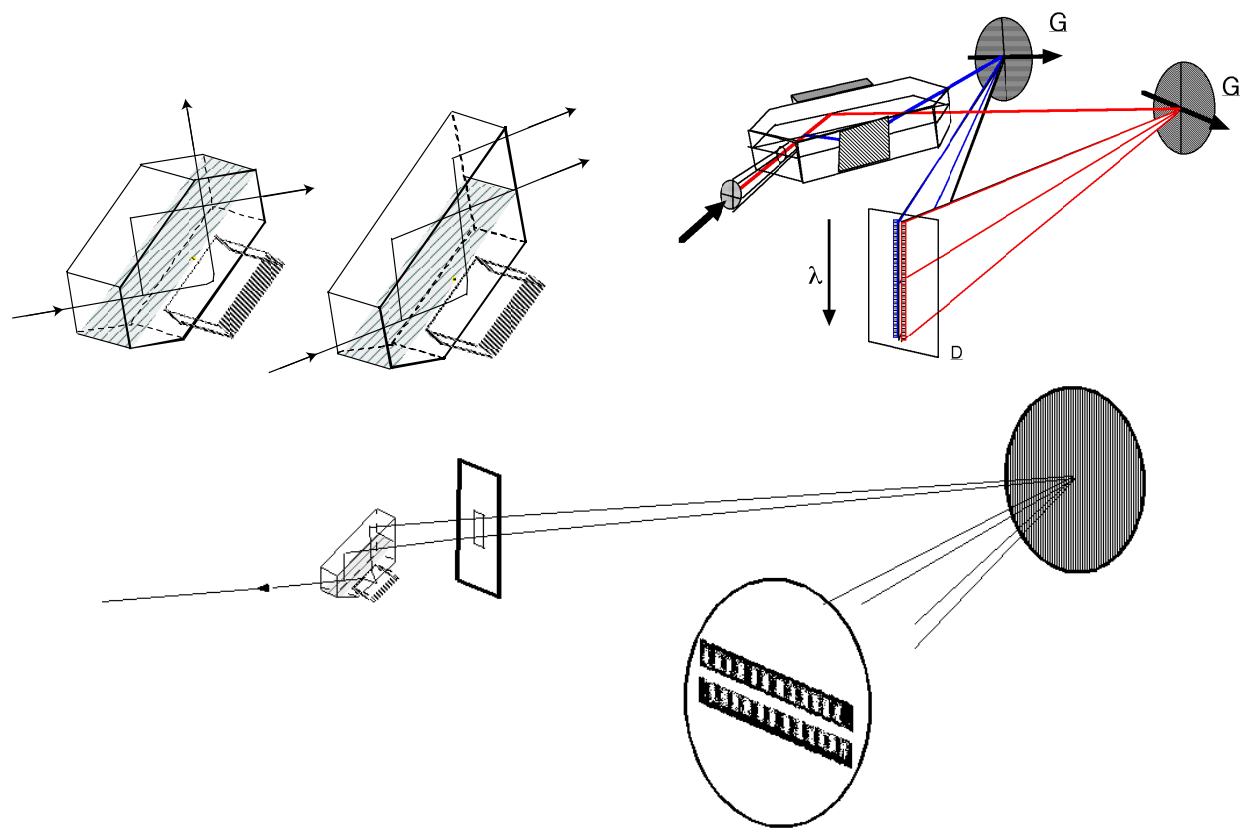

Figure 7. Complementary Output Monoliths- Schemes to use monolith interferometers (a), to reduce instrument volume and vacuum-optics losses, can be arranged to simultaneously record the complementary output beams (b, c). The nearparallel output scheme (c) is amenable to echelle spectrographs.
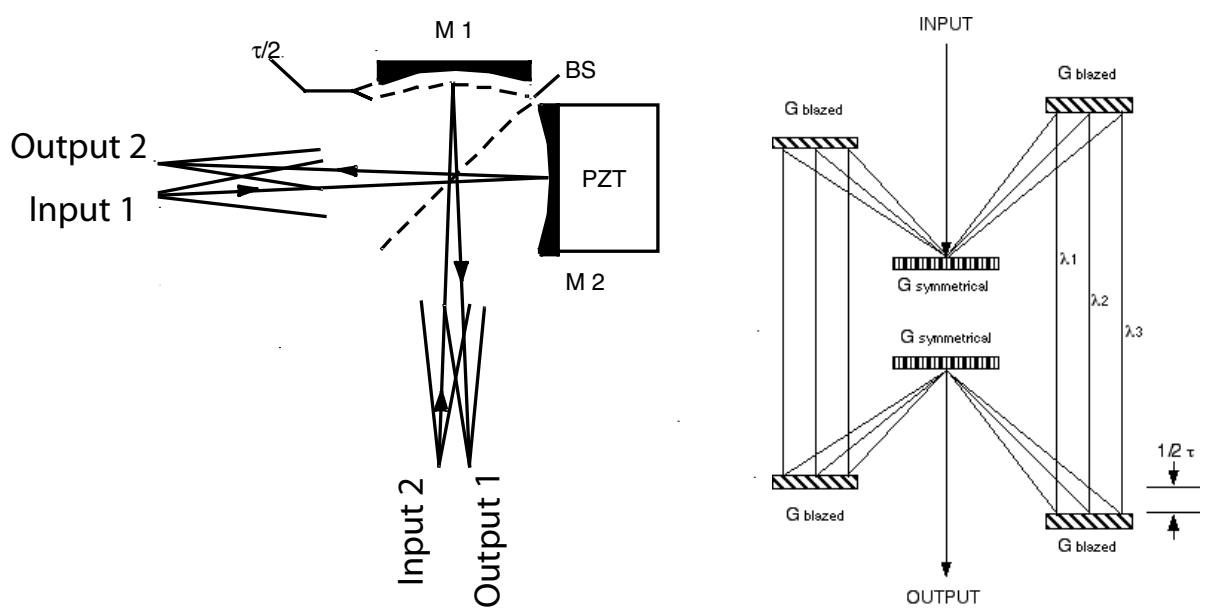

Figure 8. Reflective Interferometer Schemes- A reflective cavity Michelson scheme using a thin-plate beam splitter (a) reduce transmission losses. Completely reflective schemes (b) can use gratings as beam splitters. A symmetric profile blaze can be designed for symmetric 1st-order response and suppressed higher-orders. An asymmetric profile is used for beam turning. 
all four phase recordings. The M-Z scheme also illustrates how complementary inputs can be used to allow a simultaneous calibration source input or to allow "differential spectroscopy" where each input (e.g. star and sky) opposes the other complementary output so that the fringing data records the difference between the inputs. This differential method is familiar in FTS work and allows for simultaneous sky-subtraction or measurement of differential velocity, turbulence or rotation from adjacent fields.

A corner-mirror interferometer is shown in Fig. 6b and an off-axis Michelson scheme is shown in Fig. 6c. Other compact monolithic interferometer schemes that eliminate interface losses are shown in Fig. 7a. The complementary outputs can be collected either by a symmetric arrangement of two gratings to a single detector (Fig. 7b) or to the same grating at different imaging angles (Fig. 7c).

Beam-splitters, surfaces and solids can cause dispersion, polarization and, especially for the FUV, attenuation. To minimize these effects, all of the interferometer schemes can use thin plate splitters rather than thick path cubes. Thin (2.5 mm) $\mathrm{MgF}_{2}$ plates splitters are commercially available (Acton) with $40-50 \%$ throughput to each arm from 170-300 $\mathrm{nm}$ and 32-42\% throughput for 120-240 nm. Transmission up to $80 \%$ at $120 \mathrm{~nm}$ for a thick plate $(5 \mathrm{~mm}$ ) of LiF have been reported (Yoshino, et al. 1996). Resources in the semiconductor EUV lithograph industry are being applied to further improve LiF transmission by methods of crystal growth and contamination $\left(\mathrm{Li}_{2} \mathrm{O}\right)$ removal (Lieberman et al., 2002).

Reflective analogs of interferometer schemes mitigate transmission loss or achromatic disturbances and minimize optical interactions. Fig. 8a shows a reflective Michelson analog that uses only a thin splitter. Gratings can be used as reflective beam-splitters although simple schemes (Fig. 8b) require two symmetric-order and two blazed-order diffractions and any associated efficiency and reflectivity losses.

\section{ACKNOWLEDGMENTS}

This work was performed under the auspices of the U.S. Department of Energy by the University of California, Lawrence Livermore National Laboratory under contract No. W-7405-Eng-48, and under a CalSpace grant to the Space Sciences Lab.

\section{REFERENCES}

1. D. Erskine and J. Edelstein, "Interferometric Resolution Boosting for Spectrographs," in Ground-based Instrumentation for Astronomy, ed. A. Moorwood, SPIE Proc. 5492, June 2004.

2. D. Erskine, "An Externally Dispersed Interferometer Prototype for Sensitive Radial Velocimetry: Theory and Demonstration on Sunlight," PASP 115, pp. 255-269, 2003.

3. D. Erskine and J. Ge, "Novel Interferometer Spectrometer for Sensitive Stellar Radial Velocimetry," in Imaging the Universe in Three Dimensions: Astrophysics with Advanced Multi-Wavelength Imaging Devices, W. van Breugel and J. Bland-Hawthorn, eds., ASP 195, p. 501, 2000.

4. J. C. van Eyken, J. Ge, S. Mahadevan, and C. DeWitt, "First Planet Confirmation with a Dispersed Fixed-Delay Interferometer," ApJ 600, pp. L79-L82, Jan. 2004.

5. J. Morse, P. Scowen, M. Beasley, R. Woodruff, and HORUS MIssion Development, "The HORUS Origins Science Mission," American Astronomical Society Meeting Abstracts 205, pp. -+, Dec. 2004.

6. K. R. Sembach, D. Ebbets, R. Cen, T. Cook, R. Dave, M. Donahue, J. C. Green, E. B. Jenkins, W. R. Oegerle, J. P. Ostriker, J. X. Prochaska, B. D. Savage, J. M. Shull, H. P. Stahl, and T. M. Tripp, "The Baryonic Structure Probe: Characterizing the Cosmic Web of Matter Through Ultraviolet Spectroscopy," American Astronomical Society Meeting Abstracts 205, pp. -+, Dec. 2004.

7. D. Erskine, "Combined Dispersive/Interference Spectroscopy for Producing a Vector Spectrum," US Patent 6,351,307, Feb. 26, 2002.

8. J. Ge, D. Erskine, and M. Rushford, "An Externally Dispersed Interferometer for Sensitive Doppler Extrasolar Planet Searches," PASP 114, pp. 1016-1028, 2002.

9. D. Erskine, J. Edelstein, M. Feuerstein, and B. Welsh, "High Resolution Broadband Spectroscopy using an Externally Dispersed Interferometer," ApJ 592, pp. L103-L106, 2003. 
10. D. Erskine and J. Edelstein, "High-resolution Broadband Spectral Interferometry," in Future EUV/UV and Visible Space Astrophysics Missions and Instrumentation, ed. J. C. Blades, O. H. Siegmund, pp. 158-169, SPIE Proc. 4854, Feb. 2003.

11. N. Douglas, "Heterodyned Holographic Spectroscopy," PASP 109, p. 151, 1997.

12. S. Frandsen, N. Douglas, and H. Butcher, "An Astronomical Seismometer," A\&\&A 279, p. 310, 1993.

13. J. Harlander, R. Reynolds, and F. Roesler, "Spatial Heterodyne Spectroscopy for the Exploration of Diffuse Interstellar Emission Lines at Far-ultraviolet Wavelengths," ApJ 396, p. 730, 1992.

14. M. Born and E. Wolf, "Principles of Optics," pp. 333-338, Pergammon Press, 6th ed., 1980.

15. R. S. McMillan, T. L. Moore, M. L. Perry, and P. H. Smith, "Radial Velocity Observations of the Sun at Night," ApJ 403, pp. 801-809, Feb. 1993.

16. S. Vogt, "The Lick Observatory Hamilton Echelle Spectrometer," PASP 99, p. 1214, 1987.

17. R. Hilliard and G. Shepherd, "Wide-Angle Michelson for Measuring Doppler Line Widths," J. Opt. Soc. Am. 56, p. 362, 1966. 